\title{
Estacionalidad Reproductiva de la Hembra Bufalina (Bubalus bubalis)
}

\author{
Reproductive Seasonality of Female Buffalo (Bubalus bubalis)
}

Jorge Alberto Sánchez ${ }^{1,2}$, Marlyn Hellen Romero ${ }^{1}$, Yirly Johanna Suárez Vela ${ }^{1}$

\section{Resumen}

El objetivo de este estudio fue describir la estacionalidad reproductiva en la búfala en condiciones del trópico bajo en el Magdalena Medio colombiano y su relación con parámetros climáticos. Se evaluaron registros de nacimientos de 30571 animales desde 1970 a 2013 del Fondo Bufalero del Centro y los datos hidrometeorológicos de la estación Puerto Berrio-Antioquia, Colombia. Para el análisis, se utilizó una regresión de Poisson y una correlación de Spearman para determinar la relación entre la época de la concepción y nacimientos, expresados en porcentajes, con las siguientes variables hidrometeorológicas: valores mínimos, medios y máximos mensuales de caudales de ríos $\left(\mathrm{m}^{3} / \mathrm{seg}\right)$, valores medios, máximo mensuales de niveles de los ríos $(\mathrm{cm})$, valores totales mensuales de brillo solar $\left({ }^{\circ} \mathrm{C}\right)$, valores mínimos, medios y máximos mensuales de temperatura $\left({ }^{\circ} \mathrm{C}\right)$, valores medios mensuales de humedad relativa (\%), valores máximos mensuales de precipitación (mm en $24 \mathrm{~h}$ ), valores totales mensuales de precipitación ( $\mathrm{mm}$ ) y el índice de temperatura y humedad (ITH). El porcentaje de concepción fue mayor durante el primer trimestre del año y la mayor probabilidad de partos se presentó durante el segundo semestre. Se encontró una correlación negativa $(\mathrm{p}<0.05)$ entre la concepción y los valores máximos mensuales de caudales de río, valores media mínimos mensuales y mínimos mensuales de temperatura, así como una correlación positiva con los valores medios mensuales de humedad relativa y los valores máximos mensuales de precipitación. Se encontró una relación negativa entre el ITH y los nacimientos y la concepción. Los resultados demuestran que el búfalo en Colombia presenta ciclos estrales durante todo el año, con una estacionalidad relacionada con la temperatura ambiente, el régimen de lluvias y la humedad relativa.

Palabras clave: búfalo; estacionalidad; nacimientos; reproducción

\footnotetext{
${ }^{1}$ Laboratorio de Endocrinología y Reproducción Animal, Departamento de Salud Animal, Grupo CIENVET, Programa Medicina Veterinaria y Zootecnia, Facultad de Ciencias Agropecuarias, Universidad de Caldas, Manizales, Colombia

${ }^{2}$ Email: jorge.sanchez@ucaldas.edu.co Financiado por COLCIENCIAS y VIP Universidad de Caldas
}

Recibido: 29 de mayo de 2016

Aceptado para publicación: 7 de abril de 2017 
The aim of this study was to describe the reproductive seasonality in buffalo under tropical conditions in the Middle Magdalena, Colombia and its relation to climate parameters. Birth records of 30571 animals from 1970 to 2013 and hydro-meteorological data from Puerto Berrio-Antioquia station were evaluated. For the analysis a Poisson regression and Spearman correlation was used to determine the relationship between the season of conception and births (expressed in percentage) with the following hydrometeorological variables: minimum, average and maximum monthly flow of rivers $\left(\mathrm{m}^{3} /\right.$ $\mathrm{sec}$ ), mean values, maximum monthly river levels $(\mathrm{cm})$, monthly total values of sunshine $\left({ }^{\circ} \mathrm{C}\right)$, minimum, average and maximum monthly temperature $\left({ }^{\circ} \mathrm{C}\right)$, mean monthly values of relative humidity (\%), monthly maximum values of precipitation ( $\mathrm{mm}$ in $24 \mathrm{~h}$ ), monthly totals of precipitation $(\mathrm{mm})$ and the temperature and humidity index (THI). Conception rate was higher during the first quarter of the year and the increased likelihood of births occurred during the second half. There was a negative correlation $(p<0.05)$ between conception and the maximum monthly values of river flows, minimum monthly average values of temperature and monthly minimum temperature values. Also, a positive correlation with the monthly average values of relative humidity and maximum monthly precipitation values. There was a negative relationship between ITH with births and conceptions. The results showed that the buffalo in Colombia has estrous cycles throughout the year, with a seasonality related with temperature, rainfall and relative humidity.

Key words: buffalo; seasonality; births; reproduction

\section{INTRODUCCIÓN}

La eficiencia reproductiva es uno de los principales factores que afectan la productividad de la hembra bufalina, la cual está determinada por factores como el inicio tardío de la pubertad, la estacionalidad reproductiva multifactorial, la pobre expresión del estro y el anestro posparto prolongado con el consecuente aumento del intervalo entre partos (Murugavel et al., 2009; Nam, 2010; Sah et al., 2010; Perera, 2011). No obstante, si bien la estacionalidad reproductiva limita la producción de la especie bufalina (Baruselli et al., 1996; Martínez et al., 2009; Hassan et al., 2010), en las zonas ecuatoriales, la principal limitante de la reproducción es la nutrición y el estrés térmico (Almaguer-Pérez, 2007; Perera, 2008, 2011).
El búfalo es una especie poliéstrica que exhibe una estación reproductiva de día corto, similar a rumiantes como la oveja y la cabra (Baki et al., 2013). Su actividad sexual aumenta o es favorecida cuando las horas de luz diaria disminuyen y el clima se torna más frío (Marai y Haebb, 2010; Baki et al., 2013). En regiones tropicales como Sri Lanka, India, Cuba y la región amazónica del Brasil, donde el fotoperiodo es constante, los cambios en las precipitaciones anuales parecen influir en el patrón reproductivo, atribuido a la calidad y disponibilidad del forraje (Almaguer-Pérez, 2007; Perera, 2008, 2011), permitiendo los nacimientos en épocas de abundante disponibilidad de forraje, asegurando la producción de leche por parte de la madre y el crecimiento de los recién nacidos en condiciones climáticas favorables (Malpaux et al., 2001; Zerbe et al., 2012). 
En época de no estacionalidad reproductiva, las búfalas presentan una baja actividad sexual, que se manifiesta por una menor manifestación del estro, ciclos estrales iregulares, bajas tasas de concepción y disfunción del cuerpo lúteo $(\mathrm{CL})$, además de un aumento en la mortalidad embrionaria (Campanile et al., 2005; Rossi et al., 2014).

Estudios indican que indican que la estacionalidad reproductiva de los búfalos, bovinos y pequeños rumiantes se encuentra influenciada por el fotoperiodo (Zicarelli, 1997). Esta señal es traducida por la glándula pineal en una señal hormonal gracias a la secreción de melatonina (altos niveles por la noche, bajos niveles durante el día), la cual ejerce una acción indirecta en la secreción pulsátil de la hormona liberadora de gonadotropina $(\mathrm{GnRH})$ por medio de un complejo circuito interneural en el hipotálamo. Este proceso incluye neuronas productoras de dopamina, serotonina y neuronas aminoacidérgicas (Malpaux et al., 2001; Zarazaga et al., 2003; Caracangiu, 2011; Luridiana et al., 2012). En forma similar, el fotoperiodo puede afectar la reproducción al desinhibir la secreción activa de la hormona luteinizante $(\mathrm{LH})$ cuando aumenta la duración del día, debido a que la secreción tónica de LH es la responsable, junto con la hormona folículo estimulante (FSH), de la secreción de estradiol por parte de los folículos maduros, afectando así la ovulación (Malpaux et al., 2001; Crudeli, 2011; Di Francesco et al., 2012; Sertu et al., 2012).

El búfalo, por naturaleza, no soporta bien el calor, puesto que su cuerpo absorbe radiación solar en gran cantidad debido a su piel oscura, tamaño corporal y a su pobre capacidad de sudoración (Marai y Haeeb, 2010; Vale, 2010). El estrés generado por la alta temperatura y humedad es una limitante en la reproducción del búfalo, y se convierte en una causa de anestro importante, lo cual, asociado a las concentraciones elevadas de prolactina sanguínea, influyen en la actividad ovárica al suprimir la secreción de gonadotropinas y reducir las concentraciones de progesterona (Roy y Prakash, 2007; Das y Khan, 2010). A este fenómeno se le denomina «anestro de verano» en regiones ecuatoriales. De la misma manera, el verano afecta la foliculogenesis y la calidad de los ovocitos (Das y Khan, 2010; Nam, 2010; Di Francesco et al., 2011).

En los machos, las altas temperaturas pueden deprimir la libido, que se acompaña de una reducción en las concentraciones de testosterona y de la calidad del eyaculado (Andrabi, 2009; Nam, 2010). Además, se reporta la pérdida de la viabilidad espermática y de fertilidad durante el proceso de criopreservación (Ferrer et al., 2010; Khawaskar et al., 2012; Ibrahim et al., 2013).

Los primeros búfalos traídos a Colombia fueron ejemplares Buffalypso importados por el antiguo instituto Colombiano de Reforma Agraria Incora desde Trinidad y Tobogo en 1967. Esta raza es el producto del cruce de las razas Jaffarabadi, Nili-Ravi, Bhadawari y Surti. El nombre buffalypso que surgió al combinar la palabra búfalo con un ritmo originario de la isla, el calypso (Ángel-Marin et al., 2010; Fonseca, 2016). Los animales de esta raza se caracterizan por ser de triple propósito (carne, leche y trabajo) (ÁngelMarin et al., 2010). Actualmente, Colombia cuenta con una población cercana a los 235000 animales, distribuidos en 2672 predios, concentrados en los departamentos de Córdoba (24.8\%), Antioquia (20.4\%) y Santander (12.6\%), los cuales agrupan el $57.8 \%$ de la población nacional (ICA, 2015). Debido a que en Colombia es limitada la información sobre la estación reproductiva del búfalo, el objetivo del presente estudio fue describir la estacionalidad reproductiva en la especie bajo las condiciones de trópico bajo en el Magdalena Medio colombiano y su posible relación con parámetros hidrometeorológicos. 


\section{Materiales y Métodos}

\section{Localización}

El estudio se realizó en la región del Magdalena Medio colombiano, hacienda La Suiza, propiedad del Fondo Bufalero del Centro, localizada en los municipios de Puerto Berrio y Puerto Nare, Antioquia, Colombia. El clima de la zona se caracteriza por ser una selva húmeda tropical, con temperatura promedio de $27^{\circ} \mathrm{C}$, media anual de precipitación $2016 \mathrm{~mm}$, con una máxima de precipitación en mayo y una mínima en enero. La humedad relativa media es de $85 \%$ y su fisiografía corresponde a un relieve ligeramente ondulado con pendientes entre 3 y $12 \%$, con pendientes cortas y una altimetría entre los 125 y 200 msnm (Gobernación de Antioquia, 2005).

\section{Descripción del Estudio}

Se realizó un estudio descriptivo retrospectivo con los registros de los nacimientos de 30571 búfalos de raza Trinitaria entre 1970 a 2013, y que se encuentran registrados en el software Inter Herd ${ }^{\circledR}$ del Fondo Bufalero del Centro. Estos registros se utilizaron para el estudio de la frecuencia de tiempo y establecer la estacionalidad reproductiva.

Los datos hidrometeorológicos mensuales y anuales del Instituto de Hidrología Meteorología y Estudios Ambientales (IDEAM) se obtuvieron de la estación Puerto BerrioAntioquia (Estación 23097030 Pto Berrio), representativa de la región del Magdalena Medio colombiano y adyacente al centro bufalero. Para estimar el efecto de las variables climatológicas sobre la reproducción se utilizaron solo los registros que tenían tanto la fecha de nacimiento del animal como todas las variables climatológicas en estudio $(\mathrm{n}=351)$. Las variables evaluadas se presentan en el Cuadro 1.

El cálculo inicial de calórico parte de la propuesta de Valtorta y Gallardo (1996) y utilizada por Ospina y Camacho (2008) y Ospina
Cuadro 1. Descripción de las variables evaluadas en el estudio

\begin{tabular}{lc}
\hline Variable & Unidades \\
\hline - Nacimientos & miles, \% \\
- Concepción & miles, \% \\
- Caudal mínimo mensual & $\mathrm{m}^{3} / \mathrm{seg}$ \\
- Caudal máximo mensual & $\mathrm{m}^{3} / \mathrm{seg}$ \\
- Caudal medio mensual & $\mathrm{m}^{3} / \mathrm{seg}$ \\
- Nivel medio mensual & $\mathrm{cm}$ \\
- Nivel máximo mensual & $\mathrm{cm}$ \\
- Brillo solar total mensual & ${ }^{\circ} \mathrm{C}$ \\
- Temperatura media mensual & ${ }^{\circ} \mathrm{C}$ \\
- Temperatura media mínima & ${ }^{\circ} \mathrm{C}$ \\
mensual & \\
- Humedad relativa media & ${ }^{\circ} \%$ \\
mensual & \\
- Temperatura mínima & ${ }^{\circ} \mathrm{C}$ \\
mensual & \\
- Temperatura máxima & ${ }^{\circ} \mathrm{C}$ \\
mensual & \\
- Temperatura media-máxima & ${ }^{\circ} \mathrm{C}$ \\
mensual & \\
- Precipitación máxima & $\mathrm{mm} / 24 \mathrm{~h}$ \\
mensual & \\
- Valores totales mensuales de & \\
precipitación & $\mathrm{mm}$ \\
\hline
\end{tabular}

et al. (2012), en la que ITH $=(1.8 \mathrm{x}$ $\left.\mathrm{BS}^{\circ} \mathrm{C}+32\right)-(0.55-(0.55 \times \mathrm{HR} / 100)) \times(1.8 \times$ $\left.\mathrm{BS}^{\circ} \mathrm{C}-26\right)$, donde ITH: Índice de Temperatura Humedad, $\mathrm{BS}^{\circ} \mathrm{C}$ : Temperatura $\left({ }^{\circ} \mathrm{C}\right)$ medida por un termómetro de bulbo seco, y HR: Porcentaje de Humedad Relativa. Cuando el ITH se presenta en 70 o menos se considera que no hay condiciones ambientales para que haya estrés calórico, de 71 a 74 hay condiciones ambientales para que haya indicios de estrés calórico, de 75 a 78 las condiciones ambientales están dadas para que los animales manifiesten estrés calórico, de 79 a 83 las condiciones ambientales son extremas, los animales están lejos de su temperatura de confort y presentarán estrés calórico intenso, en tanto que superior a 83 se declara emergencia por estrés calórico. 
Cuadro 2. Frecuencia mensual de nacimientos y de concepciones de búfalas del Magdalena Medio colombiano (1970-2013)

\begin{tabular}{lcccc}
\hline \multirow{2}{*}{ Mes } & \multicolumn{2}{c}{ Concepción } & \multicolumn{2}{c}{ Nacimientos } \\
\cline { 2 - 5 } & $(\mathrm{n})$ & $(\%)$ & $(\mathrm{n})$ & $\%$ \\
\hline Enero & 4,076 & 13.3 & 2,827 & 9.3 \\
Febrero & 3,625 & 11.9 & 1,476 & 4.8 \\
Marzo & 3,548 & 11.7 & 1,162 & 3.8 \\
Abril & 2,827 & 9.3 & 1,199 & 3.9 \\
Mayo & 1,476 & 4.8 & 1,597 & 5.2 \\
Junio & 1,162 & 3.8 & 2,230 & 7.3 \\
Julio & 1,199 & 3.9 & 2,562 & 8.4 \\
Agosto & 1,597 & 5.2 & 2,928 & 9.6 \\
Septiembre & 2,230 & 7.3 & 3,341 & 10.9 \\
Octubre & 2,562 & 8.4 & 4,076 & 13.3 \\
Noviembre & 2,928 & 9.6 & 3,625 & 11.9 \\
Diciembre & 3,341 & 10.9 & 3,548 & 11.7 \\
\hline Total & 30,571 & 100.0 & 30,571 & 100.0 \\
\hline
\end{tabular}

\section{Análisis Estadístico}

Se realizó un estudio de las frecuencias de tiempo para ordenar los parámetros por mes y por año, con el fin de establecer la estacionalidad reproductiva. Se hizo una retrocuenta de 10 meses para definir la época de monta. Se elaboró una regresión de Possion para determinar la probabilidad de nacimientos y de la concepción de acuerdo al mes del año evaluado. El modelo de la regresión de Poisson fue:

$r_{j}={ }_{\breve{Y}} \beta o+\beta 1 X 1 j-\ldots+\beta k X k j$

donde $r_{j}$ es la probabilidad de que ocurra la variable dependiente (concepción/nacimientos) $\beta_{0}$ el intercepto, $\beta 1$ los coeficientes de correlación y Xi el vector de las variables independientes incluidas en el análisis.

Para hallar la relación de las variables hidrometeorológicas con la época de la con- cepción se realizó una correlación de Spearman. En todos los análisis se consideró un valor de $\mathrm{p}<0.05$ para la significancia estadística. Los análisis se efectuaron por medio del programa estadístico STATA, v. 13.0 para Windows (STATA, Texas, EEUU).

\section{Resultados}

El $54.7 \%$ de las concepciones ocurrió durante el primer semestre del año, donde la regresión de Poisson demostró que es mayor la fertilidad en el periodo de diciembre a marzo $(47.8 \%)$ y de menor concepción en junio (3.8\%) y julio (3.9\%) (Cuadro 2).

El ITH presentó un valor de $80.4 \%$ y se muestra una correlación negativa entre los valores medios mensuales de temperatura y los nacimientos, así como entre el ITH y las variables partos y concepción $\left(\mathrm{R}^{2}=-0.35\right.$; $\mathrm{p}<0.05)$. 
Cuadro 3. Correlaciones de Spearman (Rho) entre las variables climatológicas con la presentación de concepciones y nacimientos en búfalas del Magdalena Medio colombiano (1970-2013)

\begin{tabular}{lcccc}
\hline \multirow{2}{*}{ Variables climatológicas } & \multicolumn{2}{c}{ Concepción } & \multicolumn{2}{c}{ Partos } \\
\cline { 2 - 5 } & Rho & $\mathrm{P}$ & Rho & $\mathrm{P}$ \\
\hline Máximo caudal mensual $\left(\mathrm{m}^{3} / \mathrm{seg}\right)$ & -0.237 & $0.000^{*}$ & 0.072 & 0.173 \\
Temperatura media mensual $\left({ }^{\circ} \mathrm{C}\right)$ & 0.135 & $0.012^{*}$ & -0.257 & $0.000^{*}$ \\
Temperatura media mínima mensual $\left({ }^{\circ} \mathrm{C}\right)$ & -0.129 & $0.015^{*}$ & 0.210 & $0.000^{*}$ \\
Humedad relativa media mensual $(\%)$ & -0.001 & 0.973 & 0.031 & 0.570 \\
Temperatura media mensual $\left({ }^{\circ} \mathrm{C}\right)$ & -0.103 & $0.055^{*}$ & 0.234 & $0.000^{*}$ \\
Precipitación máxima mensual $(\mathrm{mm})$ & 0.315 & $0.000^{*}$ & 0.150 & $0.004^{*}$ \\
\hline
\end{tabular}

* Correspondencia $(p<0.05)$

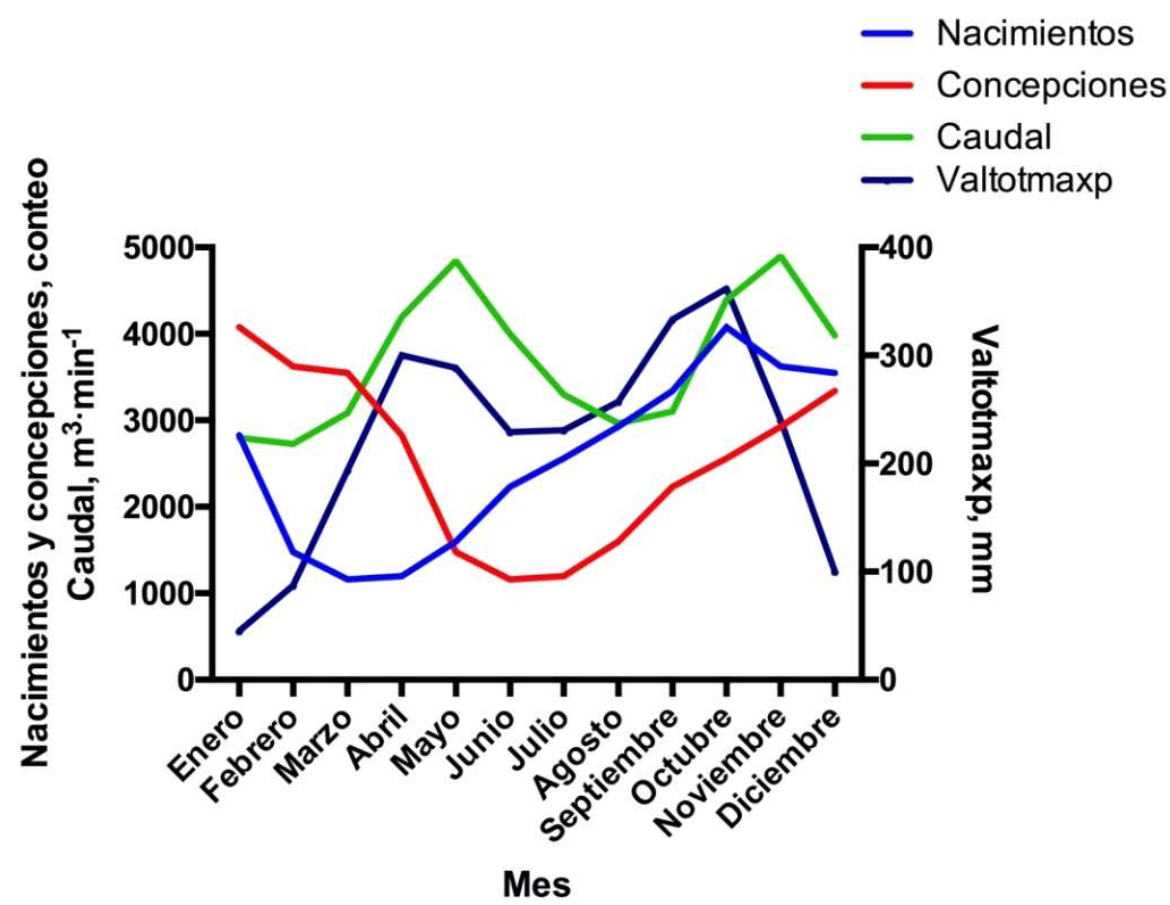

Figura 1. Relación entre nacimiento y concepción de búfalas, caudal máximo $\left(\mathrm{m}^{3} / \mathrm{seg}\right)$ del río Magdalena y valores totales máximos de precipitación (Valtotmaxp, $\mathrm{mm} / \mathrm{s}$ ) en la hacienda La Suiza (1970-2013) 


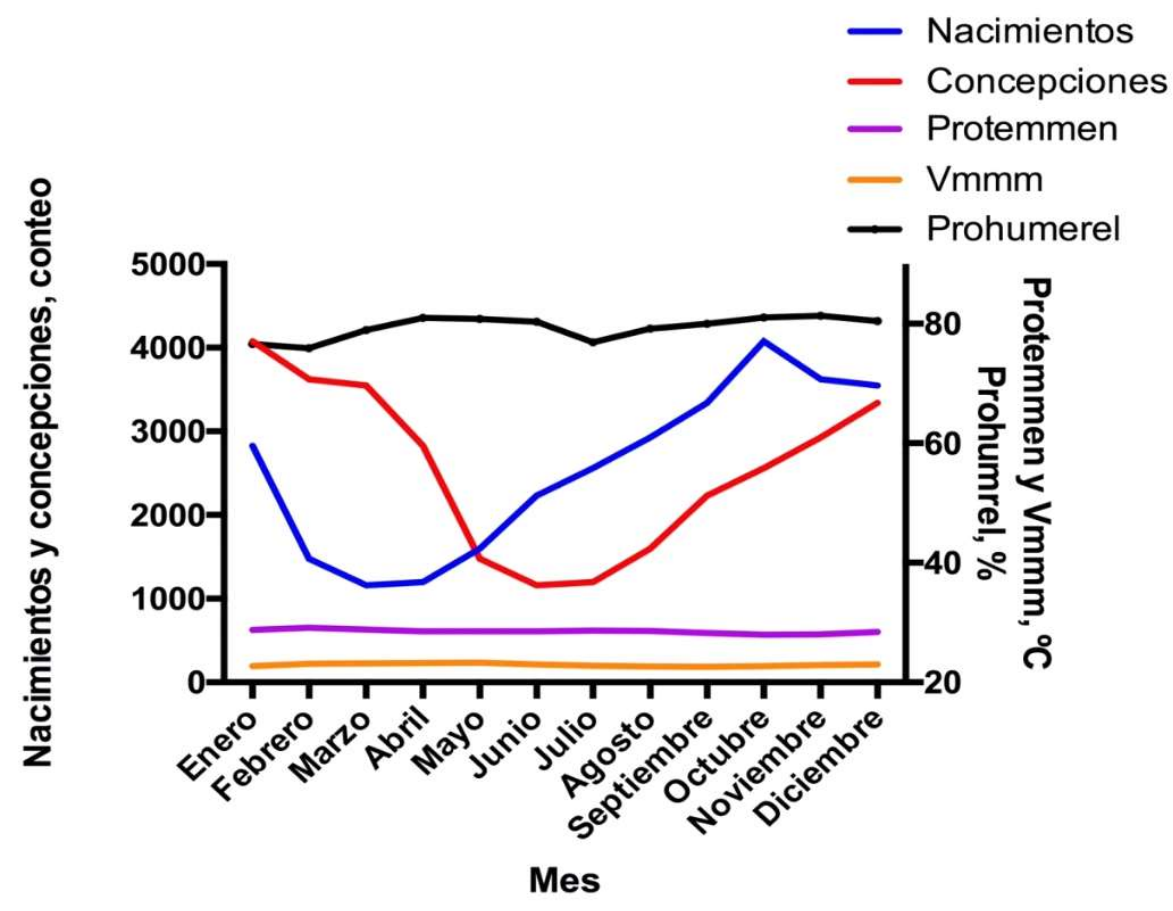

Figura 2. Relación entre nacimientos y concepción de búfalas, temperaturas medias (Protenmen) y medias mínima mensuales $\left({ }^{\circ} \mathrm{C}\right)(\mathrm{Vnmnm})$ y humedad relativa promedio mensual (\%) (Prohumerel) en la hacienda La Suiza (1970-2013)

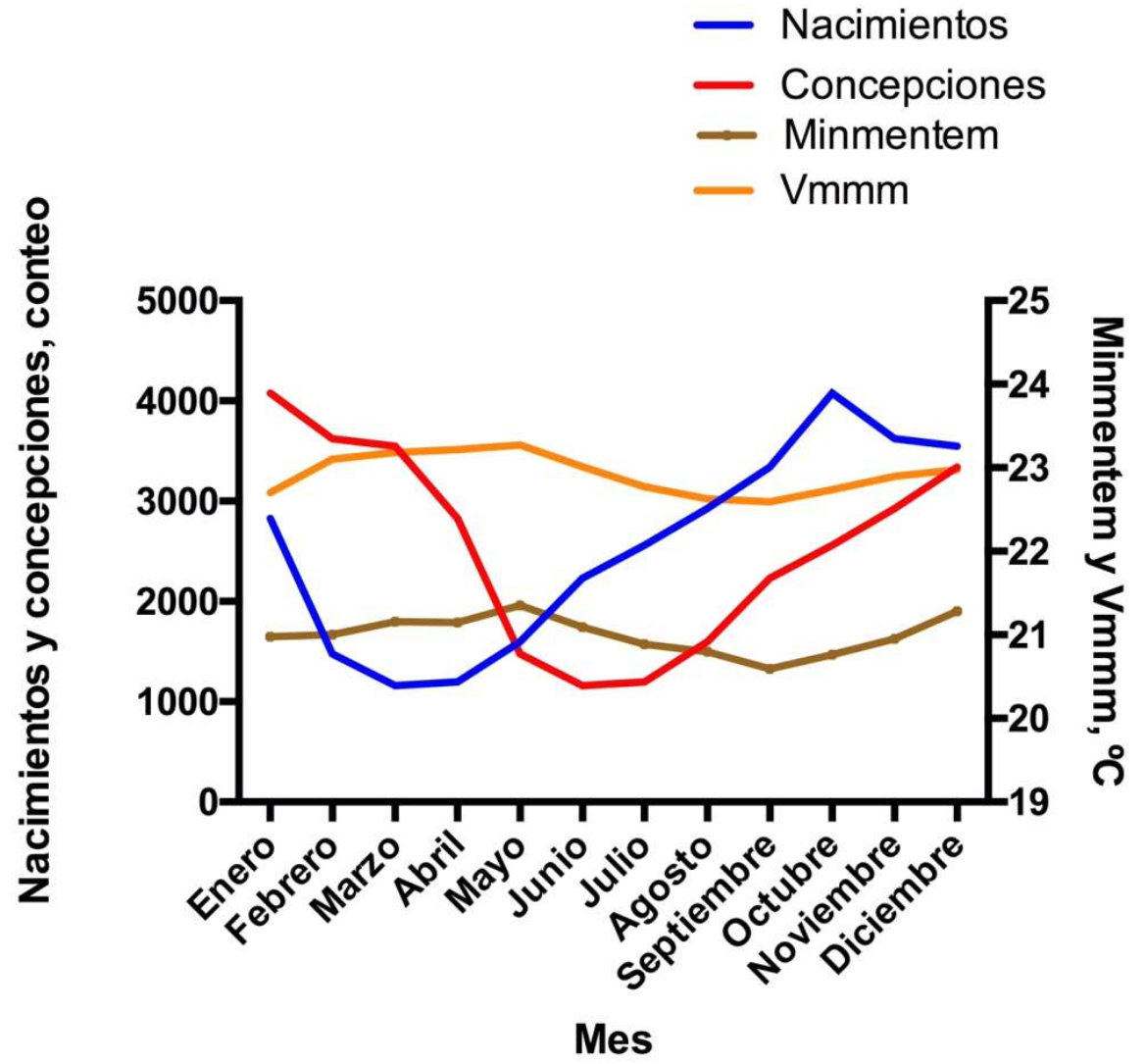

Figura 3. Relación entre nacimientos y concepción de búfalas y temperatura media mínimas (Vmmm) y mínimos mensuales $\left({ }^{\circ} \mathrm{C}\right)$ (Minmentem) en la hacienda La Suiza (1970-2013) 
Según la distribución de Poisson, se estableció que la mayor probabilidad de partos se presentó durante el segundo semestre del año $(65.7 \%)$, con un pico en octubre $(13.3 \%)$ y con la menor ocurrencia en los meses de marzo (3.8\%) y abril (3.9\%) (figuras 1,2 y 3 ).

En la correlación de Spearman se obtuvo una correspondencia positiva $(\mathrm{p}<0.05)$ entre los nacimientos y las variables valores media mínima mensuales de temperatura $\left({ }^{\circ} \mathrm{C}\right)$, valores mínimos mensuales de temperatura $\left({ }^{\circ} \mathrm{C}\right)$ y valores máximos mensuales de precipitación ( $\mathrm{mm}$ ) en $24 \mathrm{~h}$. En estos casos se observa que los partos coinciden con la segunda temporada de lluvia, en la cual aumenta los niveles del río Magdalena y se presentan las inundaciones en las vegas del río (Cuadro 3, Figura 1). Se muestra una correlación negativa entre los valores medios mensuales de temperatura y los nacimientos, así como entre el ITH y las variables partos y concepción $\left(\mathrm{R}^{2}=-0.35 ; \mathrm{p}<0.05\right)$.

Las figuras 2 y 3 presentan la relación negativa entre las concepciones y los valores máximos mensuales de caudales de río $\left(\mathrm{m}^{3} / \mathrm{seg}\right)$, valores media mínimo mensuales de temperatura $\left({ }^{\circ} \mathrm{C}\right)$ y los valores mínimos mensuales de temperatura $\left({ }^{\circ} \mathrm{C}\right)$, así como una correspondencia positiva con los valores medios mensuales de humedad relativa y los valores máximos mensuales de precipitación (mm) (Cuadro 3). Se observa una menor tasa de concepción cuando los valores medios de temperatura aumentan y, por lo tanto, el porcentaje de humedad relativa es mayor. Las demás variables hidrometeorológicas no mostraron correspondencia en la correlación de Spearman.

\section{Discusión}

El mayor número de nacimientos se registró en el segundo semestre del año (65.7\%), lo que concuerda con investigaciones realizadas en Colombia por Motta-Giraldo et al. (2014) en el departamento del Caquetá, y por Bedoya et al. (2002) y Angulo et al.
(2004) en el departamento de Córdoba. Baruselli (1993) indica que en el «Vale do Ribeira», Brasil, el mayor porcentaje de partos se presentó entre los meses de febrero a mayo, con un pico máximo en el mes de febrero. En el Mediterráneo, los nacimientos ocurren entre febrero y junio, indicando que hay una baja tasa de concepción en la primavera y el verano, atribuida al efecto del fotoperiodo (Barile, 2005).

La mayor época de concepción se presentó entre diciembre y marzo; sin embargo, Angulo et al. (2004) reportaron para el departamento Córdoba que el periodo de mayor concepción se presentó en los meses de septiembre a diciembre, especialmente noviembre. Asimismo, ensayos en búfalas bufalipso cubanas evidencian mayor cantidad de celos fértiles entre septiembre y noviembre (Martínez et al., 2009; Almaguer-Pérez et al., 2015).

Los resultados muestran que la frecuencia de nacimientos aumentó a medida que las variables hidrometereológicas se tornaban menos drásticas que las presentadas durante el verano. En el estudio se evidenció un patrón de lluvias bimodal entre abril a junio y entre octubre a diciembre, en el cual hay factores climáticos favorables para una buena producción de forraje que garantice las condiciones mínimas y que el individuo entre en un balance energético positivo que le permita una buena actividad hormonal (Ospina-Rivera et al., 2012). Estudios realizados por Sertu et al. (2012) en búfalos de Carpathia reportan que la época de nacimientos bajo condiciones tropicales puede estar asociado a una mayor disponibilidad de forraje en los meses de mayor precipitación.

Estudios indican que zona de termo-neutralidad (ZTN) en los bufalinos, al igual que en el Bos indicus, varía de 30 a $32{ }^{\circ} \mathrm{C}$ (Goswami y Narain, 1962; Crudelli, 2011). Por otro lado, Misra et al. (1963) y Crudelli (2011), al medir el coeficiente respiratorio, sugieren que los búfalos llegan a un límite crítico para el mecanismo de termorregulación a $\operatorname{los} 36^{\circ} \mathrm{C}$, 
siendo indispensable medidas alternativas para la disipación de calor (agua, lodo, sombra). El efecto del estrés por calor se agrava cuando la humedad ambiental es alta (ITH mayor de $75 \%$ ), evento que fue observado en este estudio ( $80.43 \%$ ), lo que trae como consecuencia una menor tasa de concepción cuando la humedad relativa fue mayor, posiblemente debido a la relación que existe entre la alta temperatura ambiental y el subsecuente impacto sobre la alimentación y el balance energético (Marai y Haeeb, 2010). El aumento de la temperatura ambiental debido al calentamiento global puede alterar el confort térmico de los búfalos, ya que estos no se adaptan por completo al cambio climático y, por lo tanto, se verían afectadas las funciones productivas y reproductivas de las búfalas (Upadhyay et al., 2012).

Se ha demostrado el efecto de la temperatura y la estacionalidad reproductiva en la búfala, evidenciando una reducción en el número y calidad de los folículos antrales, así como en su posterior maduración, fertilización y desarrollo embrionario de mórula a blastocisto (Abdoon et al., 2014). Asimismo, observaciones realizadas en ovarios recolectados en frigoríficos denotan que el número de folículos observables en la superficie del ovario es menor en verano comparado con los observados en invierno (Das y Khan, 2010; Das et al., 2013). Este fenómeno podría ser la causa de la estacionalidad de la concepción y nacimientos observada en el presente trabajo.

También se ha señalado que el estrés originado por las elevadas temperaturas en el verano aumenta los niveles de cortisol y de radicales libres produciendo infertilidad (Megahed et al., 2008), al afectar la foliculogenesis y el proceso de atresia, conllevando a la aciclia en las búfalas (Jan et al., 2014).

En el presente estudio no se observó asociación entre el efecto del brillo solar sobre los nacimientos y la concepción en la búfala colombiana, pero es posible que se conserven los receptores de la melatonina y puedan influir en la ciclicidad reproductiva (Carcangiu et al., 2011; Di Francesco et al., 2012; Luridiana et al., 2012). Asimismo, no se debe dejar de lado el rol del butoro en la estacionalidad reproductiva, ya que la morfometría testicular y de las glándulas accesorias en el búfalo quedan moderadamente reducidas en el verano $y$, en consecuencia, algunos parámetros del eyaculado pueden quedar alterados (Koonjaenak et al., 2007a,b; Arrighi et al., 2010; Khawaskar et al., 2012; Ibrahim et al., 2013).

\section{Conclusiones}

Las búfalas de la región del Magdalena Medio colombiano muestran una estacionalidad reproductiva asociada con la temperatura ambiental, el régimen de lluvias y la humedad relativa y los niveles del río Magdalena, sin descartar los factores genéticos y la disponibilidad de alimento.

\section{Agradecimientos}

Los autores agradecen al Patrimonio Autónomo Fondo Nacional de Financiamiento para la Ciencia, la Tecnología y la Innovación, Francisco José de Caldas, COLCIENCIAS, a la Vicerrectoría de Investigaciones y Posgrados de la Universidad de Caldas, al Fondo Bufalero del Centro, y al Instituto de Hidrología, Meteorología y Estudios Ambientales (IDEAM) por el apoyo económico para la realización del estudio.

\section{Literatura Citada}

1. Abdoon AS, Gabler C, Holder C, Kandil OM, Einspanier R. 2014. Seasonal variations in developmental competence and relative abundance of gene transcripts in buffalo (Bubalus bubalis) oocytes. Theriogenology 82: 1055-1067. doi: 10.1016/j.theriogenology.2014.07.008 
2. Almaguer-Pérez Y. 2007. El búfalo, una opción de la ganadería. REDVET 8(8). [Internet]. Disponible en: http:// www.veterinaria.org/revistas/redvet/ n080807/080709.pdf

3. Almaguer-Pérez Y, Font-Puente H, Bárzaga-González R. 2015. Estacionalidad de los partos en hembras bubalinas (buffalipso) de la Empresa Agropecuaria Bayamo en Granma. REDVET 16(5). [Internet]. Disponible en: http://www.veterinaria.org/revistas/ redvet/n050515/051511.pdf

4. Andrabi SMH. 2009. Factors affecting the quality of cryopreserved buffalo (Bubalus bubalis) bull spermatozoa. Reprod Domest Anim 44: 552-569. doi: 10.1111/j.1439-0531.2008.01240.x

5. Ángel Marín PA, Montoya AE, Martínez E, Cardona Cadavid $H$, Moreno Ochoa M, Cerón-Muñoz MF. 2010. Caracterización genética del búfalo colombiano. Livestock Res Rural Develop 22: 1-6.

6. Angulo A, Muñoz L, González M, Álvarez LD. 2004. Sincronización de la ovulación e inseminación artificial en búfalas a tiempo fijo. Rev MVZ Córdoba 9: 444-450.

7. Arrighi S, Bosi G, Groppetti D, Cremonesi F. 2010. Morpho- and histometric evaluations on the testis and epididymis in buffalo bulls during the different reproductive seasons. Open Anat J 2: 29-33. doi: 10.2174/ 1877609401002010029

8. Baki Acar D, Birdane MK, Dogan N, Gurler H. 2013. Effect of the stage of estrous cycle on follicular population, oocyte yield and quality, and biochemical composition of serum and follicular fluid in Anatolian water buffalo. Anim Reprod Sci 137: 8-14. doi: 10.1016/j.anireprosci.2012.12.004

9. Barile VL 2005. Improving reproductive efficiency in female buffaloes. Livest Prod Sci 92: 183-194. doi: 10.1016/ j.livprodsci.2004.06.014
10. Baruselli PS, Mucciolo RG, Visintin JA, Viana WG, Arruda RP, Madureira EH, Molero-Filho JR. 1996. Ovarian follicular dynamics during the estrus cycle in buffalo (Bubalus bubalis). Preliminary research. Ann NY Acad Sci 791: 408-411. doi: 10.1111/j.17496632.1996.tb53547.x

11. Baruselli PS, Oliveira JFS, Mendes MLM, Jorge AM, Fujii T, Palazzo JPC. 1993. Diagnóstico da bubalinocultura do Vale do Ribeira. Campinas. Doc Téc CATI 94.16 p.

12. Bedoya C, Mira T, Guarín JF, Berdugo JA. 2002. Parámetros reproductivos del búfalo de agua (Bubalus bubalis) en el sur de Córdoba. Costa Norte Colombiana. The buffalo: an alternative for animal agriculture in the third millenium. En: VI World Buffalo Congress. Venezuela.

13. Campanile G, Neglia G, Gasparrini B, Galero G, Prandi A, Di Palo R, D'Occhio MJ, Zicarelli L. 2005. Embryonic mortality in buffaloes synchronized and mated by AI during the seasonal decline in reproductive function. Theriogenology 63: 2334-2340. doi: 10.1016/j.theriogenology.2004.10.012

14. Carcangiu V, Mura MC, Pazzola M, Vacca GM, Paludo M, Marchi B, Daga C, et al. 2011. Characterization of the Mediterranean Italian buffaloes melatonin receptor 1A (MTNR1A) gene and its association with reproductive seasonality. Theriogenology 76: 419-426. doi: 10.1016/j.theriogenology.2011.02.018

15. Crudelli GA. 2011. Fisiología reproductiva del búfalo. Producción en Argentina. Tecnología en Marcha 24(5): 74-81.

16. Das GK, Khan FA. 2010. Summer anoestrus in buffalo - a review. Reprod Domest Anim 45: e483-e494. doi: 10.1111/j.1439-0531.2010.01598.x

17. Das GK, Kumawat BL, Khan FA. 2013. Ovarian follicular dynamics during estrous cycle and its aberrations during certain reproductive disorders in buffalo. Theriogenol Insight 3(1): 37-46. 
18. Di Francesco S, Boccia L, Campanile G, Di Palo R, Vecchio D, Neglia G, Zicarelli L, Gasparrini B. 2011. The effect of season on oocyte quality and developmental competence in Italian Mediterranean buffaloes (Bubalus bubalis). Anim Reprod Sci 123: 48-53. doi: 10.1016/j.anireprosci.2010.11.009

19. Di Francesco S, Neglia G, Vecchio D, Rossi P, Russo M, Zicarelli L, D'Occhio MJ, Campanile G. 2012. Influence of season on corpus luteum structure and function and AI outcome in the Italian Mediterranean buffalo (Bubalus bubalis). Theriogenology 78: 1839-1845. doi: 10.1016/j.theriogenology.2012.07.022

20. Ferrer A, Montes I, Alcalá Y. 2010. Influencia de la época del año en las características del semen fresco del búfalo de agua. Cienc Tecnol Ganadera 4(1): 53-58.

21. Fonseca P. 2016. Conozca las razas de búfalos que existen en Colombia. Bogotá: Contextoganadero. [Internet]. Disponible en: http://www.contexto ganadero. com/ganaderia-sostenible/conozca-lasrazas-de-bufalos-que-existen-encolombia

22. Gobernación de Antioquia. 2005. Anuario estadístico de Antioquia 2005. Departamento Administrativo de Planeación, Medellín, Colombia. [Internet]. Disponible en: http:// antioquia.gov.co/index.php/estadisticase-indicadores

23. Goswani SB, Narain P. 1962. The effect of air temperature and relative humidity on some physiological indices of buffalo-bulls (Bubalus bubalis). Indian J Vet Sci 32: 112-118.

24. Hassan F, Khan MS, Rehman MS, Sarwar M, Bhatti SA. 2010. Seasonality of calving in Nili-Ravi buffaloes, purebred Sahiwal and crossbred cattle in Pakistan. Ital J Anim Sci 6: 1298-1301. doi: 10.4081/ ijas.2007.s2.1298
25. Ibrahim N, Al-sahaf M, Alwan A. 2013. Reproductive activity of mature Iraqi bull buffaloes: testes dimensions and histological picture. Int J AnimVeter Adv 5(1): 34-37.

26. [ICA] Instituto Colombiano Agropecuario. 2015. Colombia: censo pecuario nacional - 2015. [Internet]. Disponible en: http://www.ica.gov.co/ getdoc/8232c0e5-be97-42bd-b07b9cdbfb07fcac/Censos-2008.aspx

27. Jan MH, Das GK, Khan FA, Singh J, Bashir ST, Khan S, Prasad JK, et al. 2014. Evaluation of follicular oxidantantioxidant balance and oxidative damage during reproductive acyclicity in water buffalo (Bubalus bubalis). Asian Pacific J Reprod 3: 35-40. doi: 10.1016/ S2305-0500(13)60182-7

28. Khawaskar M, Panchal MT, Dhami AJ, Hadiya KK, Patel SB. 2012. Seasonal variation in seminal biochemical constituents in Surti buffalo bulls. Indian J Anim Reprod 33: 41-46.

29. Koonjaenak S, Pongpeng P, Wirojwuthikul S, Johannisson A, Kunavongkrit A, Rodriguez-Martinez H. 2007a. Seasonality affects postthaw plasma membrane intactness and sperm velocities in spermatozoa from Thai AI swamp buffaloes (Bubalus bubalis). Theriogenology 67: 14241435.

30. Koonjaenak S, Johannisson A, Pongpeng $P$, Wirojwuthikul $S$, Kunavongkrit A, Rodriguez-Martinez H. 2007b. Seasonal variation in nuclear DNA integrity of frozen-thawed spermatozoa from Thai AI swamp buffaloes (Bubalus bubalis). J Vet Med A Physiol Pathol Clin Med 54: 377-383.

31. Luridiana S, Mura MC, Pazzola M, Paludo M, Cosso G, Dettori ML, Bua $S$, et al. 2012. Association between melatonin receptor 1A (MTNR1A) gene polymorphism and the reproductive performance of Mediterranean Italian buffaloes. Reprod Fertil Dev 24: 983987. doi: 10.1071/RD11297 
32. Malpaux B, Migaud M, Tricoire H, Chemineau P. 2001. Biology of mammalian photoperiodism and the critical role of the pineal gland and melatonin. J Biol Rhythms 16: 336-347. doi: 10.1177/074873001129002051

33. Marai IFM, Haeeb AAM. 2010. Buffalo's biological functions as affected by heat stress - a review. Livest Sci 127: 89-109. doi: 10.1016/j.livsci.2009.08.001

34. Martínez A, Ray J, López R, Benítez D, Guevara O. 2009. Comportamiento de algunos indicadores productivos y reproductivos del búfalo de río en la provincia Granma. Rev Cubana Cienc Agric 43: 127-130.

35. Megahed GA, Anwar MM, Wasfy SI, Hammadeh ME. 2008. Influence of heat stress on the cortisol and oxidantantioxidants balance during oestrous phase in buffalo-cows (Bubalus bubalis): thermo-protective role of antioxidant treatment. Reprod Domest Anim 43: 672-677. doi: 10.1111/j.14390531.2007.00968.x

36. Misra MS, Sengupta BP, Roy A. 1963. Physiological reactions of buffalo cows maintained in two different housing conditions during summer months. Indian J Dairy Sci 16: 203-215.

37. Motta-Giraldo JL,Waltero-García I, Abeledo-García MA, Miranda I, Campos-Pipaon R. 2014. Principales trastornos reproductivos en búfalas y vacas en hatos mixtos y de una especie en el departamento de Caquetá, Colombia. Rev Fac Med Vet Zoot 61: 228-240. doi: 10.15446/rfmvZ.v61n3.46870

38. Murugavel K, Antoine D, Raju MS, López-Gatius F. 2009. The effect of addition of equine chorionic gonadotropin to a progesterone-based estrous synchronization protocol in buffaloes (Bubalus bubalis) under tropical conditions. Theriogenology 71: 1120-1126. doi: 10.1016/j.theriogenology.2008.12.012

39. Nam NH. 2010. Characteristics of reproduction of the water buffalo and techniques used to improve their reproductive performance. J Sci Dev 8: 100-110.
40. Ospina-Rivera OF, Camacho Quiroga RE, Jiménez Rodríguez A, Bernal JL. 2012. Sistema de soporte de decisiones. Bogotá, Colombia: FEDEGÁN-FNG, SENA. $56 \mathrm{p}$.

41. Ospina-Rivera OF, Camacho Quiroga $R E$. 2008. Propuesta método cálculo de estrés calórico para ganado bovino en Colombia. Bogotá, Colombia: FEDEGAN-FNG OMVE. $54 \mathrm{p}$.

42. Perera BMAO. 2008. Reproduction in domestic buffalo. Reprod Domest Anim 43 (Suppl 2): 200-206. doi: 10.1111/ j.1439-0531.2008.01162.x

43. Perera ВМAO. 2011. Reproductive cycles of buffalo. Anim Reprod Sci 124: 194-199. doi: 10.1016/j.anire-prosci.2010.08.022

44. Rossi P, Vecchio D, Neglia G, Di Palo $R$, Gasparrini B, D'Occhio MJ, Campanile G 2014. Seasonal fluctuations in the response of Italian Mediterranean buffaloes to synchroni-zation of ovulation and timed artificial insemination. Theriogenology 82: 132-137. doi: 10.1016/j.theriogenology.-2014.03.005

45. Roy KS, Prakash BS. 2007. Seasonal variation and circadian rhythmicity of the prolactin profile during the summer months in repeat-breeding Murrah buffalo heifers. Reprod Fertil Dev 19: 569-575. doi: 10.1071/RD06093

46. Sah SK, Nakao T. 2010. A clinical study of anestrus buffaloes in southern Nepal. J Reprod Dev 56: 208-211. doi: 10.1262/ jrd.09-137T

47. Sertu S, Voiculescu M, Pop A, Bota A, Paraschivescu M. 2012. Research concerning the reproduction seasonality in Carpathian buffalo. Sci Papers Anim Sci Biotecnol 45: 253-258.

48. Upadhyay RC, Ashutosh, Rani R, Singh SV, Mohanty TK, Gohain M. 2012. Impact of climate change on reproductive functions of Murrah buffaloes. J Anim Plant Sci 22: 234-236.

49. Vale W. 2010. Effects of environment on buffalo reproduction. Ital J Anim Sci: 130-142. doi: 10.4081/ijas.2007.s2.130 
50. Valtorta S, Gallardo M. 1996. El estrés por calor en producción lechera. Instituto Nacional de Tecnología Agropecuaria Argentina. Miscelánea 81: 173-185.

51. Zarazaga L, Guzmán J, Pérez M. 2003. Estacionalidad reproductiva en caprino y su relación con las concentraciones plasmáticas de melatonina. Seoc 218-221.
52. Zerbe P, Clauss M, Codron D, Bingaman Lackey L, Rensch E, Streich JW, Hatt JM, Müller DW. 2012. Reproductive seasonality in captive wild ruminants: implications for biogeographical adaptation, photoperiodic control, and lifehistory. Biol Rev Camb Philos Soc 87: 965-990. doi: 10.1111/j.1469-185X.2012.-00238.x

53. Zicarelli L. 1997. Reproductive seasonality in buffalo. Bubalus Bubalis 4: 29-52. 